\title{
IDEMPOTENT-GENERATED REGULAR SEMIGROUPS
}

\author{
C. EBERHART ${ }^{1}$ W. WILLIAMS and L. KINCH
}

(Received 21 September 1970)

Communicated by G. B. Preston

Suppose $S$ is a regular semigroup and $E$ is its set of idempotents. If $E$ is subsemigroup of $S$, then $S$ has been called orthodox and studied recently by Hall [3], Meakin [6], and Yamada [8]. In this paper we assume that $E$ is not (necessarily) a subsemigroup of $S$ and consider the subsemigroup generated by $E$, denoted $\langle E\rangle$. If $E^{n}$ denotes the set of all elements of $S$ which can be written as the product of $n$ (not necessarily distinct) idempotents of $S$, then $\langle E\rangle=\bigcup_{n=1}^{\infty} E^{n}$. We show that $\langle E\rangle$ is always a regular subsemigroup of $S$ and investigate relationships between it and $S$. The case where $\langle E\rangle=S$ is of particular interest to us; such semigroups will be referred to as idempotent-generated regular semigroups.

Throughout $S$ will denote a regular semigroup and $E$ its set of idempotents. The set of inverses of an element $x$ of $S$ is denoted by $V(x)$. The regularity of $S$ guarantees that each of its elements has at least one inverse. For other facts about regular semigroups see Clifford and Preston [2].

\section{The regularity of $\langle E\rangle$}

Our first lemma was motivated by a lemma of Howie and Lallement [4].

Lemma 1.1. Let $S$ be a regular semigroup and let $n>1$. An element $x$ of $S$ can be written as the product of $n$ idempotents of $S$ if, and only if, $x$ has an inverse which can be written as the product of $n-1$ idempotents of $S$. In symbols,

$$
x \in E^{n} \Leftrightarrow V(x) \cap E^{n-1} \neq \varnothing .
$$

Proof. $\Rightarrow$ : The case $n=2$ is due to Howie and Lallement [4]. If $x=e_{1} e_{2}$, then choose $y \in V(x)$ and let $f=e_{2} y e_{1}$. One computes that $f \in V(x) \cap E$.

Inductively, suppose $n$ is a positive integer $>1$ and suppose it has been shown

${ }^{1}$ This author was supported by National Science Foundation Grant Number 21079. 
that each element of $E^{n}$ has an inverse in $E^{n-1}$. Let $x \in E^{n+1}$, say $x=e_{1} e_{2} \cdots e_{n+1}$. Pick $g \in V\left(e_{2} \cdots e_{n+1}\right) \cap E^{n-1}$ and $y \in V(x)$ and let $f=g\left(e_{2} \cdots e_{n+1} y e_{1}\right) \in g E$. By computation, one sees that $f \in V(z) \cap E^{n}$; hence, the first implication is established.

$\Leftarrow$ : Suppose $x \in S$ such that $V(x) \cap E^{n-1} \neq \varnothing$ for some positive integer $n>1$. If $n=2$, pick $e \in V(x) \cap E$ and note that $x=x e x=(x e)(e x) \in E^{2}$. If $n>2$, choose $w \in V(x) \cap E^{n-1}$ and use the first implication to choose $y \in V(w) \cap E^{n-2}$. Then

$$
x=x w x=(x w) y(w x) \in E \cdot E^{n-2} \cdot E=E^{n} .
$$

This concludes the proof of 1.1 .

COROLlary 1.2. If $S$ is a regular semigroup, then the subsemigroup $\langle E\rangle$ of $S$ generated by the idempotents of $S$ is also regular. In fact, if $x \in E^{n}$, then $V(x) \subseteq E^{n+1}$; so that every inverse of an element of $\langle E\rangle$ is also in $\langle E\rangle$.

Proof. The first assertion follows from the first implication of 1.1 . To prove the second assertion, let $y \in V(x)$. Then $x \in V(y)$ and $x \in E^{n}$; hence by the second part of 1.1, $y \in E^{n+1}$.

A natural equivalence relation to define on any regular semigroup $S$ is the following:

$x \mathscr{V} y \Leftrightarrow$ there exists a sequence $x_{1}, \cdots, x_{n}$ of elements of $S$ such that $x_{1} \in V(x)$, $x_{i} \in V\left(x_{i-1}\right)$ for $i=2, \cdots, n$ and $y \in V\left(x_{n}\right)$.

Note that $\mathscr{V}$ is simply the transitive closure of the 'inverse' relation on $S$ $(x \sim y \Leftrightarrow x \in V(y))$. Note also that $\mathscr{V} \subseteq \mathscr{D}(\mathscr{D}$ is the usual Green's relation on $S$ ) since all inverses of an element of $S$ are $\mathscr{D}$-related to that element.

The next theorem gives a description of $\langle E\rangle$ in terms of the relation $\mathscr{V}$.

THEOREM 1.3. Let $S$ be regular with idempotents $E$. Let $x \in S$. Then

(i) $x \in\langle E\rangle \Leftrightarrow$ there is an $e \in$ such that $x \mathscr{V} e$.

(ii) $x \in E^{n} \Rightarrow x \in\left(D_{x} \cap E\right)^{n+2}$.

Proof. (i) $\Rightarrow$ : Let $x \in\langle E\rangle$, and let $n$ be a positive integer such that $x \in E^{n}$. If $n=1$, the conclusion holds. If $n>1$, repeated application of the first part of 1.1 yields a sequence $x_{1}, x_{2}, \cdots, x_{n-1}$ of elements of $S$ such that $x_{1} \in V(x) \cap$ $E^{n-1}$ and $x_{i} \in V\left(x_{i-1}\right) \cap E^{n-1}$ for $i=2, \ldots, n-1$. Note that $x_{n-1} \in E$ and $x \mathscr{V} x_{n-1}$.

$\Leftarrow$ : Suppose $x \in S$ and $x \mathscr{V} e$ for some $e \in E$. Choose a sequence $x_{1}, \cdots, x_{n}$ in $S$ such that $x_{1} \in V(x), x_{i} \in V\left(x_{i-1}\right)$ for $i=2, \cdots, n$ and $e \in V\left(x_{n}\right)$. Note that $x=x x_{1} x=x x_{1} x_{2} \cdots x_{n} e x_{n} \cdots x_{2} x_{1} x$. If $n$ is odd, $x=\left(x x_{1}\right)\left(x_{2} x_{3}\right) \cdots\left(x_{n-1} x_{n}\right) e\left(x_{n} x_{n-1}\right)$ $\cdots\left(x_{3} x_{2}\right)\left(x_{1} x\right) \in E^{n+2}$. If $n$ is even, $x=\left(x x_{1}\right)\left(x_{2} x_{3}\right) \cdots\left(x_{n} e\right)\left(e x_{n}\right) \cdots\left(x_{3} x_{2}\right)\left(x_{1} x_{2}\right)$ $\in E^{n+2}$.

This concludes the proof of (i). The proof of (ii) follows upon noting that all of the products $x x_{1}, x x_{2} x_{3}, \cdots$ are in the $\mathscr{D}$-class of $x$. 
COROLlaRY 1.4. If $S$ is an idempotent-generated regular semigroup and I is an ideal of $S$, then $I$ is also an independent-generated regular semigroup.

Proof. This follows from 1.3(ii) and the fact that $I$ is the union of all the $\mathscr{D}$-class of $S$ which it meets.

\section{Green's $\mathscr{D}$-relation on $\langle E\rangle$ and a characterization of idempotent-generated regular semigroups}

It is well known that, in general, Green's relations are not well-behaved relative to subsemigroups; that is to say, if $T$ is a subsemigroup $S$, then it it not necessarily the case that $K_{T}=K_{S} \cap(T \times T)$, where $K_{S}$ denotes one of Green's relations on $S$ and $K_{T}$ denotes the corresponding relation on $T$.

In case $T$ is regular, it follows from a lemma of Anderson, Hunter, and Koch [1] that the relations $\mathscr{R}, \mathscr{L}$, and $\mathscr{H}$ do behave nicely in the above sense. However, it still may not be the case that $\mathscr{D}_{T}=\mathscr{D}_{S} \cap(T \times T)$; for example, let $S$ be the bicyclic semigroup and $T$ be its semilattice of idempotents.

In this section we shall describe the $\mathscr{D}$-relation in $\langle E\rangle$ when $S$ is a regular semigroup and use this to characterize those regular semigroups which are idempotent generated.

LEMMA 2.1. Let $T$ be a regular subsemigroup of semigroup $S$. Then

(i) $K_{T}=K_{S} \cap(T \times T)$ for $K=\mathscr{L}, \mathscr{R}$, or $\mathscr{H}$.

(ii) $x \mathscr{D}_{T} y \Leftrightarrow$ there exists $z \in T$ such that $x \mathscr{R}_{s} z \mathscr{L}_{S} y$.

The proof of (i) follows immediately from [1], whereas (ii) is a direct consequence of (i) together with the definition of $\mathscr{D}$. It will thus be necessary to denote the $\mathscr{D}$-relation on $\langle E\rangle$ by $\mathscr{D}_{\langle E\rangle}$, while the relations $\mathscr{L}, \mathscr{R}$, and $\mathscr{H}$ on $\langle E\rangle$, being restrictions of the corresponding relations on $S$, will not be subscripted.

In order to facilitate the statement of the next lemma we define the relation $\alpha$ on $E$ as follows:

$e \alpha f \Leftrightarrow$ there is a sequence $g_{1}, \cdots, g_{n}$ in $E$ such that $e K_{i} g_{1}, g_{i} K_{i+1} g_{i+1}$ for $i=2, \cdots, n-1$ and $g_{n} K_{n+1} f$, where for each $i, K_{i}$ is one of $\mathscr{L}$ or $\mathscr{R}$.

Note that $\alpha$ is simply the transitive closure of the symmetric reflexive relation

$$
[\mathscr{L} \cap(E \times E)] \cup[\mathscr{R} \cap(E \times E)]
$$

and is thus an equivalence. Note also that $\alpha \subseteq \mathscr{V}$ since if two idempotents are $\mathscr{L}$ or $\mathscr{R}$ related they are inverses of one another. Roughly speaking, two idempotents are $\alpha$-related if it is possible to get from one to the other by going along the $\mathscr{R}$ and $\mathscr{V}$ classes of $S$, turning corners only when one is an $H$-class containing an idempotent.

LEMMA 2.2. Let $S$ be regular with idempotents $E$. Then $\alpha=\mathscr{D}_{\langle E\rangle} \cap(E \times E)$. 
PRoOF. $\subset:$ Since $\mathscr{R}_{\langle E\rangle} \cup \mathscr{L}_{\langle E\rangle} \subseteq \mathscr{D}_{\langle E\rangle}$ and since $\alpha$ is the transitive closure of $[\mathscr{L} \cap(E \times E)] \cup[\mathscr{R} \cap(E \times E)]$, the containment follows.

$\supset$ : We say that a pair $(x, y) \in \mathscr{D}_{\langle E\rangle} \cap(E \times E)$ has degree $n$ provided $n$ is the smallest positive integer $k$ for which there exists $z \in E_{k}$ such that $x \mathscr{L} z \mathscr{R} y$. Let $(e, f) \in \mathscr{D}_{\langle E\rangle} \cap(E \times E)$. Then clearly if the degree of $(e, f)$ is 1 , then $e \alpha f$. Inductively, suppose that $n$ is a positive integer for which it has been shown that whenever a pair of idempotents $\left(e^{\prime}, f^{\prime}\right)$ is of degree not exceeding $n$, then $e^{\prime} \propto f^{\prime}$. Now if the degree of $(e, f)$ is $n+1$, pick $z \in E^{n+1}$ such that $e \mathscr{L} z \mathscr{R} f$. By 1.1, pick $z^{\prime} \in V(z) \cap E^{n}$, and let $e^{\prime}=z z^{\prime}, f^{\prime}=z^{\prime} z$. Note that $e^{\prime}, f^{\prime} \in E$, $f \mathscr{R} z \mathscr{R} e^{\prime}$ and $f^{\prime} \mathscr{L} z \mathscr{L} e$; hence $f \mathscr{R} e^{\prime} \mathscr{L} z^{\prime} \mathscr{R} f^{\prime} \mathscr{L} e$. By the induction hypothesis, since $f^{\prime}$ and $e^{\prime}$ have degree at most $n, e^{\prime} \alpha f^{\prime}$; hence $e \propto f$. Since the pair $(e, f)$ must have some degree, this completes the proof of the containment.

THEOREM 2.3. Let $S$ be a regular semigroup with idempotents $E$. Then $\mathscr{D}_{\langle E\rangle}=\mathscr{V} \cap(\langle E\rangle \times\langle E\rangle)$.

Proof. Suppose $(x, y) \in \mathscr{V} \cap(\langle E\rangle \times\langle E\rangle)$. Choose $x_{1}, x_{2}, \cdots, x_{n}$ in $S$ so that $x_{1} \in V(x), x_{i} \in V\left(x_{i-1}\right)$ for $i=2, \cdots, n$, and $y \in V\left(x_{n}\right)$. Now since $x \in\langle E\rangle$, it follows from 1.2 that $x_{1} \in\langle E\rangle$, and hence $x \mathscr{D}_{\langle E\rangle} x_{1}$. In the same way we find $x_{1} \mathscr{D}_{\langle E\rangle} x_{2}$, and hence $x \mathscr{D}_{\langle E\rangle} x_{2}$. Continuing in this manner we finally conclude that $x \mathscr{D}_{\langle E\rangle} y$.

Conversely suppose $x \mathscr{D}_{\langle E\rangle} y$. By 1.3(i), we can choose $e, f \in E$ such that $x \mathscr{V} e$ and $y \mathscr{V} f$. It follows from the first part of this proof that $x \mathscr{D}_{\langle E\rangle} e$ and $y \mathscr{D}_{\langle E\rangle} f$; hence $e \mathscr{D}_{\langle E\rangle} f$. Thus by $2.2, e \alpha f$ and so $e \mathscr{V} f$. Hence $x \mathscr{V} y$ and the equality is established.

As a corollary we obtain the following characterization of idempotentgenerated regular semigroups.

COROLlaRY 2.4. A regular semigroup $S$ is idempotent-generated if, and only if, $\mathscr{D}=\mathscr{V}$.

Proof. $\Rightarrow$ : This implication is immediate by setting $S=\langle E\rangle$ in 2.3.

$\nLeftarrow$ : Suppose $S \neq\langle E\rangle$. By 1.3(i), there is an element whose $\mathscr{V}$-class contains no idempotent. Since every $\mathscr{D}$-class contains at least one idempotent, it follows that $\mathscr{Z} \neq \mathscr{V}$.

The next theorem describes when the $\mathscr{D}$-relation on $\langle E\rangle$ is well-behaved in the sense that $\mathscr{D}_{\langle E\rangle}=\mathscr{D}_{\mathrm{S}} \cap(\langle E\rangle \times\langle E\rangle)$.

THEOREM 2.5. For a regular semigroup $S$, the following are equivalent:

(i) $a=\mathscr{D}_{S} \cap(E \times E)$

(ii) $\mathscr{D}_{\langle E\rangle}=\mathscr{D}_{S} \cap(\langle E\rangle \times\langle E\rangle)$

(iii) Each $\mathscr{H}$-class of $S$ contains a product of idempotents $S$. 
Proof. (i) $\Rightarrow$ (ii): By 2.1, $\left.\mathscr{D}_{\langle E\rangle} \subseteq \mathscr{D}_{S} \cap(\langle E\rangle) \times\langle E\rangle\right)$. If $(x, y) \in \mathscr{D}_{S} \cap(\langle E\rangle$ $\times\langle E\rangle$ ), choose idempotents $e$ and $f$ such that $e \mathscr{L} x$ and $y \mathscr{R} f$. Then $e \mathscr{D} s$ and so $(e, f) \in \mathscr{D}_{S} \cap E \times E$ and hence using (i) $e \alpha f$. But by $2.2 \alpha=\mathscr{D}_{\langle E\rangle} \cup(E \times E)$ hence $e \mathscr{D}_{\langle E\rangle} f$. So $x \mathscr{L}_{e} \mathscr{D}_{\langle E\rangle} f \mathscr{R} y$ from which we get that $x \mathscr{D}_{\langle E\rangle} y$.

(ii) $\Rightarrow$ (iii): Let $H$ be an $\mathscr{H}$-class of $S$. Then $H=R \cap L$ where $R$ is an $\mathscr{R}$-class of $S$ and $L$ is an $\mathscr{L}$-class of $S$. Choose $e \in R \cap E$ and $f \in L \cap E$. So $e \mathscr{D}_{S} f$ and hence using (ii) $e \mathscr{D}_{\langle E\rangle} f$. Thus there is a $z \in\langle E\rangle$ such that $e \mathscr{R} z \mathscr{L} f$ and thus $z \in H \cap\langle E\rangle$.

(iii) $\Rightarrow$ (i): We always have $\alpha \subseteq \mathscr{D}_{S} \cap(E \times E)$. If $(e, f) \in \mathscr{D}_{S} \cap(E \times E)$ then by (iii) there is a $z \in\left(R_{e} \cap L_{f}\right) \cap\langle E\rangle$. Hence $(e, f) \in \mathscr{D}_{\langle E\rangle} \cap(E \times E)$ and so by $2.2, e \alpha f$. This concludes the proof.

\section{The simple case}

If $S$ is a simple (no proper ideals) regular semigroup with idempotents $E$, then of course it need not be that $\langle E\rangle$ is simple; for example take $S$ to be the bicycle semigroup.

In this section we shall first investigate the relationship between $S$ and $\langle E\rangle$ when one of them is simple.

Lemma 3.1. Let $S$ be a regular semigroup. Then

(i) $S$ is simple if, and only if, for every e, $f \in E$, there is $a n a \in S$ and an inverse $a^{\prime}$ of $a$ such that $a a^{\prime}=e$ and $a^{\prime} a f=a^{\prime} a$.

(ii) $S$ is bisimple (one $\mathscr{D}$-class) if, and only if, for each $e, f \in E$ there is an $a \in S$ an inverse $a^{\prime}$ of $a$ such that $a a^{\prime}=e$ and $a^{\prime} a=f$.

Proof. These statements are proved for inverse semigroups in Clifford and Preston [2,II] and no essential use is made of the uniqueness of inverses.

THEOREM 3.2. Let $S$ be a regular semigroup. Then

(i) If $\langle E\rangle$ is simple, then $S$ is simple

(ii) If $S$ is simple and each $\mathscr{H}$-class of $S$ meets $\langle E\rangle$, then $\langle E\rangle$ is simple.

(iii) $\langle E\rangle$ is bisimple if, and only if, $S$ is bisimple and each $\mathscr{H}$-class of $S$ meets $\langle E\rangle$.

(iv) $\langle E\rangle$ is completely simple if, and only if, $S$ is completely simple.

Proof. (i) follows from 3.1(i).

(ii) let $e, f \in E$. By 3.1(i), choose $a \in S$ and $a^{\prime} \in V(a)$ such that $a a^{\prime}=e$ and $a^{\prime} a f=a^{\prime} a$.

Now choose $x \in H_{a} \cap\langle E\rangle$, where $H_{a}$ denotes the $\mathscr{H}$-class of $a$, and let $x^{\prime}$ denote the inverse of $x$ which lies in $H_{a^{\prime}}$. Then we have $x x^{\prime}=a a^{\prime}=e$ and $x^{\prime} x f=a^{\prime} a f=a^{\prime} a=x^{\prime} x$. Since by 1.2, $x^{\prime} \in\langle E\rangle$, we conclude from 3.1(i) that $\langle E\rangle$ is simple. 
(iii) If $\langle E\rangle$ is bisimple, then $S$ is bisimple from 3.1(ii). Now if $H$ denotes an $\mathscr{H}$-class of $S$, let $e, f \in E$ such that $R_{e} \cap L_{f}=H$. Since $\left.e \mathscr{D}_{\langle E\rangle}\right\rangle$ such that $e \mathscr{R} z \mathscr{R} f$; hence $z \in H \cap\langle E\rangle$.

(iii) If $\langle E\rangle$ is completely simple, then $\langle E\rangle$ has a primitive idempotent; hence $S$ has a primitive idempotent. By 3.2(i), $S$ is simple. Hence $S$ is completely simple. Conversely if $S$ is completely simple, then $S$ is simple and each $\mathscr{H}$-class of $S$ meets $\langle E\rangle$; hence by 3.1(ii), $\langle E\rangle$ is simple. But also $S$ has a primitive idempotent which must also then be a primitive idempotent of $\langle E\rangle$, so $\langle E\rangle$ is completely simple.

REMARK 3.3. The authors have so far been unable to find an example of a simple idempotent-generated regular semigroup which is not completely simple. The following theorem seems to indicate that possibly no such example exists.

THEOREM 3.4. The only simple regular semigroup with identity which is idempotent-generated is the one element semigroup.

Proof. Let $S$ denote such a semigroup and let $e$ denote the identity of $S$. First we shall show that $E^{n} \cap L_{e}=\{e\}=E^{n} \cap R_{e}, n=1,2, \cdots$ : so suppose $f \in E \cap L_{e}$. Then $x f=e$ for some $x \in S$, so $e=x f=(x f) f=f$. Hence $E \cap L_{e}=\{e\}$. Similarly $E \cap R_{e}=\{e\}$. Inductively, suppose that $n$ is a positive integer for which it has been shown that $E^{n} \cap L_{e}=\{e\}=E^{n} \cap R_{e}$. Let $x \in$ $x \in E^{n+1} \cap L_{e}$. By 1.1, there exist $y \in V(x) \cap E^{n}$. Moreover, since $E \cap L_{x}=$ $E \cap L_{e}=\{e\}$, it must be that $y \in R_{e}$. But by the induction hypothesis, $R_{e} \cap E^{n}=$ $\{e$,$\} hence y=e$. Hence $e=y=y x y=e x e=x$, and we conclude that $E_{n+1}$ $\cap L_{e}=\{e\}$. Similarly $E^{n+1} \cap R_{e}=\{e\}$. Since $S=\langle E\rangle$, we conclude that $L_{e}=R_{e}=\{e\}$.

Now suppose $f \in E$. Since $S$ is simple, there exists, by 3.1(i), an element $a \in S$ and an inverse $a^{\prime}$ of $a$ such that $a a^{\prime}=e$ and $a^{\prime} a f=a^{\prime} a$. But $a a^{\prime}=e$ implies that $a \in R_{e}=\{e\}$. Hence $a=e=a^{\prime}$ and so $e=a^{\prime} a=a^{\prime} a f=e e f=f$. Thus $E=\{e\}$ and the proof is complete.

Suppose $S$ is a completely simple semigroup and $E$ is the set of idempotents of $S$. One may ask about the structure of $\langle E\rangle$ beyond the fact that $\langle E\rangle$ is completely simple. The next theorem shows among other things, that $\langle E\rangle$ is determined to a large extent by the number of $\mathscr{L}$ and $\mathscr{R}$ classes of $S$.

THEOREM 3.5. Let $\alpha$ and $\beta$ be non-zero cardinal numbers and let $X$ and $Y$ be sets of cardinal $\alpha$ and $\beta$ respectively. Let $\mathfrak{F}_{X x Y}$ denote the free semigroup on $X x Y$ and let $\rho$ be the congruence on $\mathfrak{\mho}_{X x Y}$ generated by the relations $(x, y)(x, z)=(x, z)$ and $(x, y)(z, y)=(x, y)$. Denote the quotient semigroup obtained by $T(\alpha, \beta)$. Then $T(\alpha, \beta)$ is an idempotent-generated completely simple semigroup having $\beta \mathscr{L}$-classes and $\alpha \mathscr{R}$-classes. Further if $S$ is an idempotentgenerated completely simple semigroup having $\beta \mathscr{L}$-classes and $\alpha \mathscr{R}$-classes then $S$ is a homomorphic image of $T(\alpha, \beta)$. 
Proof. For $(x, y) \in X x Y$, let $[x, y]$ be the $\rho$-class of $(x, y)$. Suppose $[x, y]$ $=\left[x^{\prime}, y^{\prime}\right]$, and fix $\left(x_{0}, y_{0}\right) \in X \times Y$. Consider the mapping $\phi: X \times Y \rightarrow x_{0} \times Y$ defined by $\phi(x, y)=\left(x_{0}, y\right)$. This induces a homomorphism $\Phi$ from $T(\alpha, \gamma)$ onto $x_{0} \times Y$ with right trivial multiplication. Since $\left(x_{0}, y\right)=\bar{\phi}[x, y]=\Phi\left[x^{\prime}, y^{\prime}\right]$ $=\left(x_{0}, y^{\prime}\right)$ we conclude that $y=y^{\prime}$. Dually $x=x^{\prime}$. Also note that $[x, y]$ is idempotent. Thus $T(\alpha, \beta)$ is idempotent-generated.

$T(\alpha, \beta)$ is a union of groups: If $z=\prod_{i=1}^{n}\left[x_{1}, y_{i}\right] \in T(\alpha, \beta)$, then $z \in\left[x_{1}, y_{n}\right] T(\alpha, \beta)\left[x_{1}, y_{n}\right]$. Also one computes that

$$
z^{\prime}=\left[x_{1}, y_{n}\right]\left(\prod_{t=0}^{n-2}\left[x_{n-i}, y_{n-i-1}\right]\right)\left[x_{1}, y_{n}\right]
$$

is an inverse for $z$ in $\left[x_{1}, y_{n}\right] T(\alpha, \beta)\left[x_{1}, y_{n}\right]$. Further $z z^{\prime}=z^{\prime} z=\left[x_{1}, y_{n}\right]$, so $\left[x_{1}, y_{n}\right] T(\alpha, \beta)\left[x_{1}, y_{n}\right]$ is a group. Note also that the idempotent of each group (and thus every idempotent of $T(\alpha, \beta))$ is of the form $[x, y]$.

$T(\alpha, \beta)$ is simple: Let $a, b \in T(\alpha, \beta)$. Choose inverses $a^{\prime}$ and $b^{\prime}$ for $a$ and $b$ respectively. Then $a a^{\prime}=[x, y]$ and $b b^{\prime}=[w, z]$ for some $(x, y)$ and $(w, x)$ in $X \times Y$. Now

$$
a=a a^{\prime} a=[x, y] a=[x, z][w, z][x, y] a=[x, z] b b^{\prime}[x, y] a \in T(\alpha, \beta) b T(\alpha, \beta) .
$$

Hence $T(\alpha, \beta)$ is simple.

Thus we conclude that $T(\alpha, \beta)$ is completely simple. Now fix an idempotent $e=\left[x_{0}, y_{0}\right]$ in $T(\alpha, \beta)$. One computes that the idempotents in the same $\mathscr{R}$-class with $e$ are precisely those of the form $\left[x_{0}, y\right], y \in Y$. Hence there are $\beta \mathscr{L}$-classes A dual argument shows that there are $\alpha \mathscr{R}$-classes.

To prove the last assertion of the theorem, fix an idempotent $e$ in $S$ and consider the sets $e S \cap E$ and $E e \cap E$. By assumption these have cardinality $\beta$ and $\alpha$ respectively. Let $1-1$ onto mappings $X \stackrel{g}{\rightarrow} S e \cap E$ and $T \stackrel{f}{\rightarrow} e S \cap E$ be given and define a map $X \times Y \stackrel{h}{\rightarrow} E$ by $h(x, y)$ is the idempotent in $g(x) S \cap S f(y)$ Then clearly $h$ is $1-1$ and onto. Further one verifies that the defining relations for $\rho$ are satisfied. Hence there is a unique homomorphism $h: T(\alpha, \beta) \rightarrow\langle E\rangle$ which extends $h$. This completes the proof of 3.5 .

We remark that we have been unable in general to determine the nature of the group of $T(\alpha, \beta)$. We conjecture that it is a free group on $\gamma$ generators, where $\gamma$ is a function of $(\alpha, \beta)$. For example, if $\alpha=\beta=2$, then the group of $T(\alpha, \beta)$ can easily be shown to be the integers.

\section{References}

[1] L. W. Anderson, R. P. Hunter, and R. J. Koch, 'Some results on stability in semigroups,' Trans. Amer. Math. Soc., 117 (1965), 521-529.

[2] A. H. Clifford and G. B. Preston, The algebraic theory of semigroups, Vols I and II, Math. Surveys No. 7, (Providence, R. I., 1961 and 1967.) 
[3] J. A. Erdös, 'On products of idempotent matrices,' Glasgow Math. J., 8 (1967), 118-112.

[4] T. E. Hall, 'On regular semigroups whose idempotents form a subsemigroup', Bull. Austral. Math. Soc., 1 (1969), 195-208.

[5] J. M. Howie, 'The subsemigroup generated by the idempotents of a full transformation semigroup', J. London Math. Soc. 41 (1966), 707-716.

[6] J. M. Howie and G. Lallement 'Fundamental congruences on a regular semigroup', Proc. Glasgow Math. Assoc., Vol. 7 (1966), 145-159.

[7] G. Lallement, 'Congurences et équivalences de Green sur un demi-groupe régulier,' $C . R$. Acad. Sci. Paris., Serie A, 262 (1966), 613-616.

[8] J. Meakin, 'Congurences on orthodox semigroups', J. Australian Math. Soc. 12 (1971) 323-341.

[9] M. Petrich, 'The maximal semilattice decomposition of a semigroup,' Math.Zeit. 85 (1964), 68-82.

[10] Miyuki Yamada, 'On a regular semigroup in which the idempotents form a band', Pacific J. Math. 33 (1970), 261-272.

University of Kentucky

Lexington, Kentucky 40506

University of Louisville

Louisville, Kentucky 40208

U.S.A. 